\title{
Study of adhesion and migration dynamics in ubiquitin E3A ligase (UBE3A)-silenced SYSH5Y neuroblastoma cells by micro-structured surfaces
}

\author{
R Mezzena ${ }^{1}$, C Masciullo $^{1}$, S Antonini ${ }^{1}$, F Cremisi $^{2}$, M Scheffner $^{3}$, M Cecchini $^{1}$ \\ and I Tonazzini ${ }^{1,4}(\mathbb{C}$ \\ I NEST, Istituto Nanoscienze- CNR and Scuola Normale Superiore, Pisa, Italy \\ ${ }^{2}$ Scuola Normale Superiore, Bio@SNS, Pisa, Italy \\ ${ }^{3}$ University of Konstanz, Department of Biology, Konstanz, Germany \\ ${ }^{4}$ Fondazione Umberto Veronesi, Milano, Italy \\ E-mail: ilaria.tonazzini@sns.it
}

\begin{abstract}
During neuronal development, neuronal cells read extracellular stimuli from the micro/nano-environment within which they exist, retrieving essential directionality and wiring information. Here, focal adhesions (FAs-protein clusters anchoring integrins to cytoskeleton) act as sensors, by integrating signals from both the extracellular matrix environment and chemotactic factors, contributing to the final neuronal pathfinding and migration. In the processes that orchestrate neuronal development, the important function of ubiquitin E3A ligase (UBE3A) is emerging. UBE3A has crucial functions in the brain and changes in its expression levels lead to neurodevelopmental disorders: the lack of UBE3A leads to Angelman syndrome (AS, OMIN 105830), while its increase causes autisms (Dup15q-autism). By using nano/micro-structured anisotropic substrates we previously showed that UBE3A-deficient neurons have deficits in contact guidance (Tonazzini et al, Mol Autism 2019).

Here, we investigate the adhesion and migration dynamics of UBE3A-silenced SH-SY5Y neuroblastoma cells in vitro by exploiting nano/micro-grooved substrates. We analyze the molecular processes regulating the development of FAs by transfection with EGFPvector encoding for paxillin, a protein of FA clusters, and by live-cell total-internalreflection-fluorescence microscopy. We show that UBE3A-silenced SH-SY5Y cells have impaired FA morphological development and pathway activation, which lead to a delayed adhesion and also explain the defective contact guidance in response to directional topographical stimuli. However, UBE3A-silenced SH-SY5Y cells show an overall normal migration behavior, in terms of speed and ability to follow the GRs directional stimulus. Only the collective cell migration upon cell gaps was slightly delayed for UBE3Ash SHs. Overall, the deficits of UBE3Ash SHS-SY5Y cells in FA maturation/sensing and in collective migration
\end{abstract}


may have patho-physiological implications, in AS condition, considering the much more complex stimuli that neurons find in vivo during the neurodevelopment.

Keywords: SH-SY5Y, micrograting, contact guidance, mechanotransduction, focal adhesions, Angelman Syndrome, UBE3A

\section{Introduction}

All cells are exposed to extracellular signals determined by the local micro/nano-environment. In the nervous system, neurons grow and exert their activities while embedded in a dense environment, the extracellular matrix, which contains an array of structural and directional cues, in the dimensional range of nano-micro meters. Here, environmental sensing triggers complex intracellular signaling patterns that are integrated by cells to guide neuronal adhesion, migration, wiring and plasticity $[1,2]$.

External features (e.g. the extracellular matrix, collagen fibers, radial glia) are read by specific cellular systems, including integrin receptors and focal adhesion clusters, a process called mechanotransduction [3]. In this framework, focal adhesions (FAs; i.e. protein clusters anchoring transmembrane integrins to cytoskeleton elements) act as sensors, by integrating both physical and chemical external signals $[4,5]$. FAs increase in size as they mature, and they enrich with proteins such as paxillin and activate focal adhesion intracellular pathway (i.e. at the level of FA kinase-FAK). Ultimately, FAs establish connections with cytoskeleton fibers and mediate coordinated rearrangements of the cytoskeleton, both directly (via physical connection) and indirectly (via the activation of complex intracellular signalling pathways) [3]. FAs, thanks to the cross-talk with cytoskeleton fibers, modulate cell contractility, which is essential for both neuronal motility and functionality $[4,6]$. Overall, FAs have a primary role in establishing neuronal cell polarity, migration and neurite path-finding/navigation [7].

Nowadays biomaterial science allows direct investigation of the processes that control neuronal contact sensing by using nano-textured substrates $[8,9]$. Nano/micro-structured surfaces are capable of tuning the morpho-functional properties of neuronal cells, and can be a powerful tool to test neuronal cell dynamic response to selected physical stimuli in vitro [10].

Microgratings (GRs; anisotropic topographies composed of alternating lines of grooves and ridges with sub-millimeter lateral dimensions) induce directional topographical stimuli via pure contact interaction [11]. In fact, the topographical features (e.g. ridge size, depth) in the micron/submicron range act as physical boundaries providing a local constraint to the formation and maturation of Fas [12]. We demonstrated that GRs. according to their ridge size, can tune neuronal differentiation, polarization, and neurite orientation by interfering with the cell FA machinery $[13,14]$. Neuronal polarization and topographical guidance are based on a geometrical constraint of FAs resulting in an angular modulation of their maturation and persistence: the outcome is that only aligned adhesions are allowed to grow, while misaligned ones, blocked by the topographical constraint, cannot develop into mature FAs. Additionally, cell contractility also contributes and induces the maturation of nascent adhesions at the tip of aligned neurites, whereas those produced at the tip of misaligned processes remain immature and are eventually lost [12]. Overall, neuronal cells (e.g. NGF-differentiated PC12 cells, SHSY5Y cells, leach and murine neurons) follow the GRs and contact guidance requires Rho-associated protein kinase (ROCK)-myosin-II-activated contractility [14-16].

Although deficits in neuronal migration and microconnectivity (i.e. leading to functional connectivity deficits) are recently emerging as crucial in many cognitive disorders (e.g. autism spectrum disorders, schizophrenia) [17], the role of neuronal sensing mechanisms during migration is little investigated in pathological conditions.

During neuronal development, the role of ubiquitination (i.e. at the level of E3 ligases) recently emerged as pivotal [18]. In particular, the ubiquitin E3A ligase (UBE3A) has a key role in neurodevelopment [19]. Importantly, the exact level of UBE3A in the brain is crucial: its lack leads to Angelman Syndrome (AS; OMIN \#105 830) [20, 21], while its increase causes autisms (e.g. Dup15q syndrome) [22]. Ube3a-deficient models showed deficient synaptic plasticity, abnormalities at the level of dendritic arborization and polarity in vivo and of spine development/actin reorganization [23-27], but no mechanistic insights were proposed. Although several UBE3A ubiquitinated targets were described [28, 29], the exact role of UBE3As in neuron morphogenesis and in the pathogenesis of UBE3A-associated disorders is still unknown. Importantly, Ube $3 a$ is transcribed to form three splice variants encoding three isoforms with different cellular localization and likely function $[30,31]$. In mice, besides two UBE3A protein isoforms with ligase activity (UBE3A 2 and 3), there is one isoform (UBE3A 1) which is not transcribed to protein and has not ligase activity. The three $U B E 3 A$ mRNA transcripts differently regulate neuronal arborization in mice [31]. Remarkably, UBE3A 1 functions as a dendritic competing endogenous RNA of microRNA miR-134 (a brain-specific microRNA that regulate synaptic development and chemo-attractive response) $[31,32]$ and miR-134 acts by interfering with several adhesion/cytoskeleton signaling effectors in different cells [33]. In humans, three UBE3A isoforms exist that differ in the length of their $\mathrm{N}$-terminal extensions. Furthermore, a human UBE3A 
transcript (UBE3A-005; ENST00000604860) was reported which is a respective mRNA as the UBE3A 1 of rodents [31]. However, if this mRNA is also expressed in humans, is a matter of debate.

Thanks to GRs, we showed for the first time the aberrant morphological phenotype of AS neurons in vitro. We found that neurite contact guidance along GRs is defective in UBE3A-deficient (i.e. model of AS) hippocampal neurons at early stages of development in vitro, and this phenotype is linked to an impaired activation of the FA signalling pathway (at the level of FAK phosphorylation) [34]. We further investigated the role of UBE3A in contact guidance, finding that AS neurons have a specific deficit in axonal topographical guidance in response to GR directional stimuli in vitro, and show in parallel increased axonal branching, with more frequent main secondary axonal segments [35]. On the other side, UBE3A overexpression does not affect neuronal directional guidance along GRs [35].

Here, we investigate the adhesion and migration dynamics of UBE3A-silenced SH-SY5Y neuroblastoma cells in vitro by exploiting nano/micro-grooved substrates. We analyze the molecular processes regulating the development of FAs, by transfection with EGFP-vector encoding for paxillin and by Total-Internal-Reflection-Fluorescence microscopy in living cells, and the migration performance, at single cell and collective levels, on both standard and directional GRs substrates.

\section{Methods}

\subsection{Substrate fabrication}

GRs were fabricated by thermal nano imprinting lithography (NIL) on copolymer 2-norbornene ethylene [cyclic olefin copolymer (COC)] foils [IBIDI, Martinsried, Germany] [16], by using molds fabricated by electron beam lithography and dry etching techniques, as reported in [36]. NIL is based on the combination of pressure and heat, which aids the transfer of the chosen pattern from a rigid mold to thermoplastic materials.

We generated a set of substrates characterized by GR surface topographies with constant groove depth $(350 \mathrm{~nm}), 50 \%$ duty cycle (i.e. ridge width $=$ groove width), and period of $2 \mu \mathrm{m}$ (i.e. $1 \mu \mathrm{m}$ ridge and $1 \mu \mathrm{m}$ groove); for selected experiments we used T1-GRs, with same depth and period of $1 \mu \mathrm{m}$ (500 $\mathrm{nm}$ ridge and groove). From previous works $[16,34]$ and preliminary experiments on $\mathrm{SHs}$, we know they both induce directional stimuli and cell contact guidance. A control flat substrate (named FLAT) was also used as reference isotropic surface. The imprinted substrates were quality checked by optical microscopy and attached to the bottom of hollowed $35 \mathrm{~mm}$ Petri dishes by using silicone glue (RS Components RS692-524). Importantly, surface wettability was not affected by the imprinting procedure or by the presence of NGs. Before cell culturing, the samples were sterilized by treatment with ethanol $70 \%$ and then rinsed with $\mathrm{H}_{2} \mathrm{O}_{\mathrm{mQ}}$ twice.

\subsection{SH-SY5Y cell culture}

Human neuroblastoma-derived SH-SY5Y (SH) cells, both wild type (SHs-WT) and SHs cells in which UBE3A expression was down-regulated by RNA interference (SHs-UBE3Ash)- AS like condition, were kindly provided by Prof. M. Scheffner [37]. SHs cells were grown in DMEMF12 medium supplemented with $10 \%$ FBS, 4 mM glutamine, $10 \mathrm{U} \mathrm{ml}^{-1}$ penicillin-10 mg ml${ }^{-1}$ streptomycin, and puromycin (Sigma; $1 \mu \mathrm{g} \mathrm{ml}^{-1}$ ), and passed with Accutase (all products were from Thermo Fisher, Waltham, MA) and were maintained in standard conditions $\left(37{ }^{\circ} \mathrm{C}, 95 \%\right.$ humidity, $5 \% \mathrm{CO}_{2}$ ). Cells were cultured until sub-confluence, and then harvested for cell tests and seeded on GRs or flat substrates.

\subsection{Cell adhesion experiments}

SHs cell amount was measured by the 2-(2-methoxy4-nitrophenyl)-3-(4-nitrophenyl)-5-(2,4-disulfophenyl)-2 Htetrazolium-monosodium salt (WST-8) assay, according to instructions (Sigma, 96 992). Briefly, SHs were seeded in 96 well-plates at a density of $2 \times 10^{4}$ cells/well. After 1-3-6 h from seeding, SHs were rinsed and incubated in a $10 \%$ WST8 solution (in medium) in a $\mathrm{CO}_{2}$ incubator for $2 \mathrm{~h}$, and the absorbance of each well was observed by a plate reader at a wavelength of $450 \mathrm{~nm}$. The absorbance of formazan produced is directly proportional to the number of living cells. A standard curve was performed for each experiment. The results are reported as percentages of the adhered cells over the seeded cells. We performed $\mathrm{n} \geq 5$ independent experiments for each condition (each condition was performed in double).

\subsection{Western blot and Focal adhesion pathway activation}

Western blot analysis on SHs was performed to assess the presence and activation (phosphorylation) levels of effector proteins in the FA pathway, such as $\beta 1$-integrin, FAK, protooncogene tyrosine-protein kinase Src (SRC), and paxillin; the level of UBE3A protein was also checked. SHs-WT and-UBE3Ash were cultured on standard plates until subconfluence and then lysed on ice in RIPA buffer (SigmaAldrich, R0278) containing protease and phosphatase inhibitors cocktail (Complete and PhosSTOP, Roche Diagnostics, Basel, Switzerland). Cell lysates were centrifuged (15000 g for $25 \mathrm{~min}, 4^{\circ} \mathrm{C}$ ) and then the supernatants were tested for protein concentration by a protein assay kit (Micro BCA ${ }^{\mathrm{TM}}$, Thermo Scientific Pierce). The samples were mixed with Laemmli buffer containing $\beta$-mercapto-ethanol (5\% final concentration), boiled for $5 \mathrm{~min}$, and used for gel electrophoresis (or kept at $-80{ }^{\circ} \mathrm{C}$ ). SHs lysates $\left(30 \mu \mathrm{g} \mathrm{lane}^{-1}\right.$ ) were processed by immunoblot, as in [35]. Briefly, samples were resolved by gel electrophoresis (SDS-PAGE) using Gel Criterion XT-Precast polyacrylamide gel $4 \%-12 \%$ Bis-Tris (Biorad), transferred to nitrocellulose membranes and probed overnight at $4{ }^{\circ} \mathrm{C}$ with primary antibodies. We used the following antibodies against: FAK (1:1000; Abcam, Cambridge, UK; ab40794) and phospho(Tyr397)-FAK (1:1000; 
Abcam ab4803); paxillin (1:5000; Abcam ab32084); $\beta 1$ integrin (1:500; Sigma); actin (1:2500; Sigma-Aldrich A3853); UBE3A (1:1000; Sigma-Aldrich E8655). Membranes, after incubation with the appropriate peroxidase-linked secondary antibodies (goat anti-Rabbit/Mouse IgG-HRP Conjugate, Biorad; 1:2500), were developed by the SuperSignal West Femto (Thermo Scientific Pierce, \#34 095) or ClarityTM (Biorad, 170-5060) enhanced chemiluminescent substrates. The chemiluminescent signal was acquired by ImageQUANT LAS400 scanner (GE Healthcare Life Sciences, Uppsala, Sweden). The density of immunoreactive bands was quantified by ImageJ; the results were normalized to the total actin content, and reported in percentages in respect to SHs-WT levels. We run $\mathrm{n} \geq 7$ independent samples for each condition, and each sample was run at least twice.

\subsection{Focal adhesion experiments and total internal reflection fluorescence (TIRF) microscopy}

SHs cells were transfected with EGFP-Paxillin construct by electroporation, as previously reported [14]. EGFP-Paxillin was a kind gift from Juergen Wehland (Helmholtz Centre for Infection Research, Braunschweig, Germany). SHs cells were transfected (using $0.5 \mu \mathrm{g}$ of plasmid DNA for 150-200000 cells) using a Microporator (Neon Electroporation Transfection system, Thermo Fisher), following the instructions.

After $24 \mathrm{~h}$ from transfection, SHs cells adhering to GRs or flat COC substrates were imaged in live. Total internal reflection fluorescence (TIRF) imaging was performed using an inverted Leica AF6000 microscope with an oil immersion $63 \times 1.46$ NA TIRF objective. For each region, brightfield (focused on the nanostructure), an epifluorescence and a TIRF (with a laser penetration depth of $200 \mathrm{~nm}$ ) images were acquired.

For FA analysis, as in [14, 38], TIRF images were loaded into ImageJ (National Institute of Health, USA) and inverted. FAs were manually drawn using the 'freehand selection' tool. Then measurements of FA area (in $\mu \mathrm{m}^{2}$ ) and alignment angle versus GR were then obtained using the 'measurement' and 'angle' tools of ImageJ, respectively. The angle of each FAs was measured with respect to the GR direction choosing the cell soma center as origin; a random reference direction $\left(0^{\circ}\right)$ was chosen for the flat surfaces. FAs were considered aligned if the angle is between $0^{\circ}$ and $15^{\circ}$ and misaligned if between $15^{\circ}$ and $90^{\circ}$. The size distribution of FAs was also analyzed and FAs were categorized as small (area $\leq 2 \mu \mathrm{m}^{2}$ ), intermediate $\left(2<\right.$ area $\left.\leq 4 \mu \mathrm{m}^{2}\right)$ and large $\left(\operatorname{area}>4 \mu \mathrm{m}^{2}\right)$ and their amount reported as percentages over the total FAs number. The number of FAs per cell was also registered. We analyzed at least ten cells for each sample, and we performed $\mathrm{n} \geq 5$ independent experiments on GRs and $n=3$ on Flat, for each condition.

\subsection{Cell morphological development}

SHs neuronal guidance response to GRs directional stimuli was quantified by measuring neurite alignment along the GR direction, at $24 \mathrm{~h}$ from seeding; a minimum threshold of $10 \mu \mathrm{m}$ for neurite length was imposed for this analysis. Living-cell imaging was performed using an inverted Nikon- $t i$ PSF widefield microscope (Nikon, Japan), with a 20x NA objective and equipped with an incubated chamber coupled to the microscope (Okolab, Italy).

Morphometric data were collected using ImageJ (National Institute of Health, USA). The GR direction was measured as an angle by ImageJ angle tool; FLAT substrates were given a $0^{\circ}$ angle.

Neurites $(\geq 10 \mu \mathrm{m})$ were semi-automatically segmented (from the point of origin at the perimeter of the cell body to the tip of the neurite growth cone) using NeuronJ, a plugin of ImageJ, and analyzed in Matlab (MathWorks, Natick, MA, USA), as previously reported [38].

We analyzed at least 15 cells, and we performed $n \geq 4$ independent experiments ( $n=8$ for GRs) for each condition.

\section{7. miR-134 measurements}

SHs-WT and SHs-UBE3Ash were cultured on standard plates until sub-confluence (around $6 \mathrm{~d}$ ), then detached and counted. 6-700000 SHs cells were then pelleted, quickly frozen in nitrogen and then stored at $-80^{\circ} \mathrm{C}$. Small RNA was extracted with miRNeasy Tissue/Cells Advanced Mini kit (Qiagen) and cDNA of mature miRNAs was synthesized using miScript System kit (Qiagen), according to the manufacturer's instructions. qRT-PCR of mmu-miR-134-5p (MIMAT0000146) was performed using MesaGreen master mix (Corbett) and a Rotor-Gene 6000 machine (Corbett). Forward U6 snRNA primer: 5 '-CAAAAGTGGAGAAATAAGGCAGG- ${ }^{\prime}$; forward mmu-miR-135-5p primer: 5'-UGUGACUGGUUGACCAGAGGGG- $3^{\prime}$. Amplification take-off values were evaluated using the built-in Rotor-Gene 6000 'relative quantitation analysis' function, and relative expression was calculated with the $2^{\wedge}-\Delta \Delta \mathrm{Ct}$ method [39], normalizing to U6 snRNA. $n=4$ replicates (with two technical replicates each) were analysed for each cell type. Wilcoxon signed rank test was employed for the statistical analysis of these results.

\subsection{Single cell migration}

SHs were seeded on T1-GRs and Flat substrates at a density of $2 \times 10^{4}$ cells $\mathrm{cm}^{1}$. After $24 \mathrm{~h}$, living-cell imaging was performed using an inverted Nikon-Ti PSF wide-field microscope (Nikon, Japan) equipped with an incubated chamber coupled to the microscope (Okolab, Italy) with an air 20x (NA 0.45, Plan-Fluor) objective, for the following $24 \mathrm{~h}$, sampling every $15 \mathrm{~min}$.

Movies were analyzed with the ImageJ manual tracking plugin MTrack [40]. The following parameters were measured: migration step (dS; corresponding to the cell motion calculated in $15 \mathrm{~min}$, in $\mu \mathrm{m}$ ): step vectors were analyzed along two directions, and dS was considered parallel (dS//) if the angle between the step and the GR was between $0^{\circ}$ and $15^{\circ}$, while it was considered perpendicular (dS $\perp$ ) for angle between $75^{\circ}$ and $90^{\circ}$; the amount of parallel or perpendicular dS was reported as percentage over the dS total number. Cell displacement ( $\mathrm{R}$, the distance, in $\mu \mathrm{m}$, from the origin 
after $24 \mathrm{~h}$ ). Average cell speed ( $\mathrm{V}$, in $\mu \mathrm{m} / \mathrm{s})$; as previously for $\mathrm{dS}$, the average speed was also quantified as parallel $(\mathrm{V} / /)$ and perpendicular $(\mathrm{V} \perp)$. We analyzed at least 15 cells for each sample, and we performed $n \geq 3$ independent experiments for each condition.

\subsection{Collective migration}

SHs were seeded on GRs and Flat substrates at a density of $1 \times 10^{5}$ cells $/$ chamber $\left(0.22 \mathrm{~cm}^{2}\right)$ in Ibidi culture-inserts (IBIDI GmbH, Martinsried, Germany). After $24 \mathrm{~h}$, the insert (width of cell-free gap $=500 \mu \mathrm{m} \pm 50 \mu \mathrm{m}$ ) was removed $(t=0)$ and cell imaging was performed using an inverted Nikon- $t i$ PSF wide-field microscope, at 0-24-48-72-96 h. The gap (average size $=584 \pm 60$ and $620 \pm 48 \mu \mathrm{m}^{2}$ for WT and UBE3Ash on flat; $873 \pm 9$ and $844 \pm 87 \mu \mathrm{m}^{2}$ respectively on GRs) was perpendicular to the GR pattern. The percentage of area closure was reported. We performed $n \geq 4$ independent experiments for each condition.

\subsection{Immunostaining and confocal fluorescence microscopy}

SHs cells were fixed for $10 \mathrm{~min}$ with $4 \%$ formaldehyde $/ 4 \%$ sucrose in PBS at room temperature and processed, as previously reported [35]. The cells were incubated with anti-Tubulin-BIII (Abcam ab78078; 1:200) or anti-N-Cad antibody (BD Transduction Laboratories; 1:250) primary antibody in GDB buffer $(0.2 \%$ BSA, $0.8 \mathrm{M} \mathrm{NaCl}, 0.5 \%$ Triton $\mathrm{X}-100,30 \mathrm{mM}$ phosphate buffer, $\mathrm{pH}$ 7.4) containing Alexa Fluor ${ }^{\mathrm{TM}} 647$ Phalloidin (1:40; A22287, Thermo Fisher) to stain actin fibers, overnight at $4{ }^{\circ} \mathrm{C}$. Samples were then incubated with the appropriate Alexa488-conjugated secondary antibody (Thermo Fisher, 1:200) in GDB for $1 \mathrm{~h}$ at room temperature, washed and mounted using Vectashield mounting medium (Vector Laboratories).

Confocal images were acquired using a laser scanning confocal microscope TCS SP2 (Leica Microsystems, Germany) with a $40 x$ oil objective. Each confocal image was a $z$-series (stack-depth was around $5 \mu \mathrm{m}$; steps $=1 \mu \mathrm{m}$ ), each averaged three times, and was chosen to cover the entire region of interest from top to bottom. The resulting $z$-stack was processed by Image $\mathbf{J}$ software (NIH) into a single image using 'z-project' and 'max intensity' options.

\subsection{Statistical analysis}

Data are reported as average values \pm the standard error of the mean (mean $\pm \mathrm{SEM}$ ). All cell experiments were repeated at least three times independently for each reported dataset $(\mathrm{n} \geq 3)$. Data were statistically analyzed by GraphPad PRISM 5.00 program (GraphPad Software, San Diego, CA, USA). Student's $t$-test (unpaired, two-tailed) analysis was used, if not differently stated, to compare WT and UBE3Ash conditions. When more than two conditions were compared, oneway ANOVA (Tukey's multiple comparison test) analysis was used. The mean values obtained in repeated experiments were assumed to be normally distributed about the true mean. Statistical significance refers to $\mathrm{P}<0.05$.

\section{Results}

\subsection{Adhesion dynamics}

We checked the adhesion of SHs. WT and UBE3Ash SHs were seeded on culture plates and the amount of adhered cells was quantified after short times, 1-3-6 h (figure 1(a)). The short-term adhesion of UBE3Ash was reduced with respect to $\mathrm{WT}(\mathrm{P}<0.05$ at 1 and $3 \mathrm{~h})$ and normalized around $6 \mathrm{~h}$ post seeding.

Therefore, we measured the activation of the focal adhesions pathway at the molecular level, looking at the level of FAK phosphorylation and FA scaffold proteins presence. Western-blot results confirmed an impaired adhesion, showing a lower activation of FAK ( $\mathrm{P}<0.01$ WT vs. UBE3Ash) and a lower level of $\beta 1$-integrin $(\mathrm{P}<0.05)$ in UBE3Ash SHs (figure 1(b)). Paxillin levels were instead not changed by the UBE3A loss.

Overall, data showed that the adhesion process and the following activation of FA pathway are impaired in UBE3Ash SHs cells.

It is known that the adhesion process is mediated by the formation and maturation (i.e. enlargement) of FAs. Therefore we investigated FA dynamics in SHs cells, to check if their establishment and maturation may be compromised by UBE3A loss. SHs cells were transiently transfected with EGFP-Paxillin, a signal-transduction protein present in early and mature FAs [3], and cultured on flat and on directional GRs substrates. Paxillin was then selectively visualized at the cell basal-membrane by live cell TIRF microscopy, with optimal spatial resolution (figure 2(a)-(c)). FAs were investigated by measuring their density (number of adhesions per cell), maturation (adhesion area), and spatial distribution on GR pattern (with respect to the GR direction).

The UBE3A loss did not affect FA density, showing similar values both on isotropic Flat and on anisotropic GRs substrates (figure 2(d), (g)).

FA maturation was then analyzed on the different substrates, by measuring the FA size. As first, on flat substrates, the mean FA average size decreased by silencing UBE3A $(\mathrm{P}<0.05$ WT vs. UBE3Ash; figure 2(e)). In order to better highlight this effect, FAs were sorted in three categories (figure 2(f)): small (FA area $\leq 2 \mu \mathrm{m}^{2}$ ), intermediate $\left(2 \mu \mathrm{m}^{2}<\right.$ area $\left.\leq 4 \mu \mathrm{m}^{2}\right)$, and large $\left(>4 \mu \mathrm{m}^{2}\right)$, corresponding to different maturation stages [12]. Silencing UBE3A led to a marked increase of the small-FA population (from $20 \%$ in WT to $30 \%$ in UBE3Ash SHs; $\mathrm{P}<0.01$ WT vs. UBE3Ash), and to a reduction of large FAs (from $47 \%$ in WT to $25 \%$ in UBE3Ash; $\mathrm{P}<0.05$ WT vs. UBE3Ash).

We know that FAs act as topographical sensors on GRs and we therefore quantified the FAs spatial distribution on the pattern. FAs were analyzed by measuring their alignment $\left({ }^{\circ}\right)$ with respect to the GR direction. FAs were classified as aligned (in case of alignment angle $\leq 15^{\circ}$ ) or misaligned (in case of alignment angle $>15^{\circ}$ ). The percentage of aligned FAs dropped down from $59 \pm 3 \%$ for SHs WT to $44 \pm 2 \%$ for SHs UBE3Ash ( $\mathrm{P}<0.01$; figure $2(\mathrm{~h})$ ); as expected, a random distribution of FAs was found on isotropic flat substrates, with 


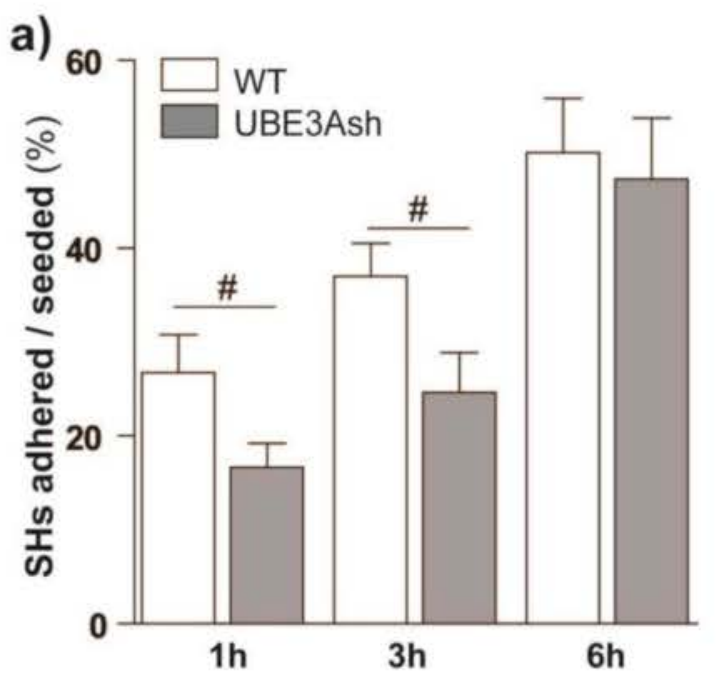

b)

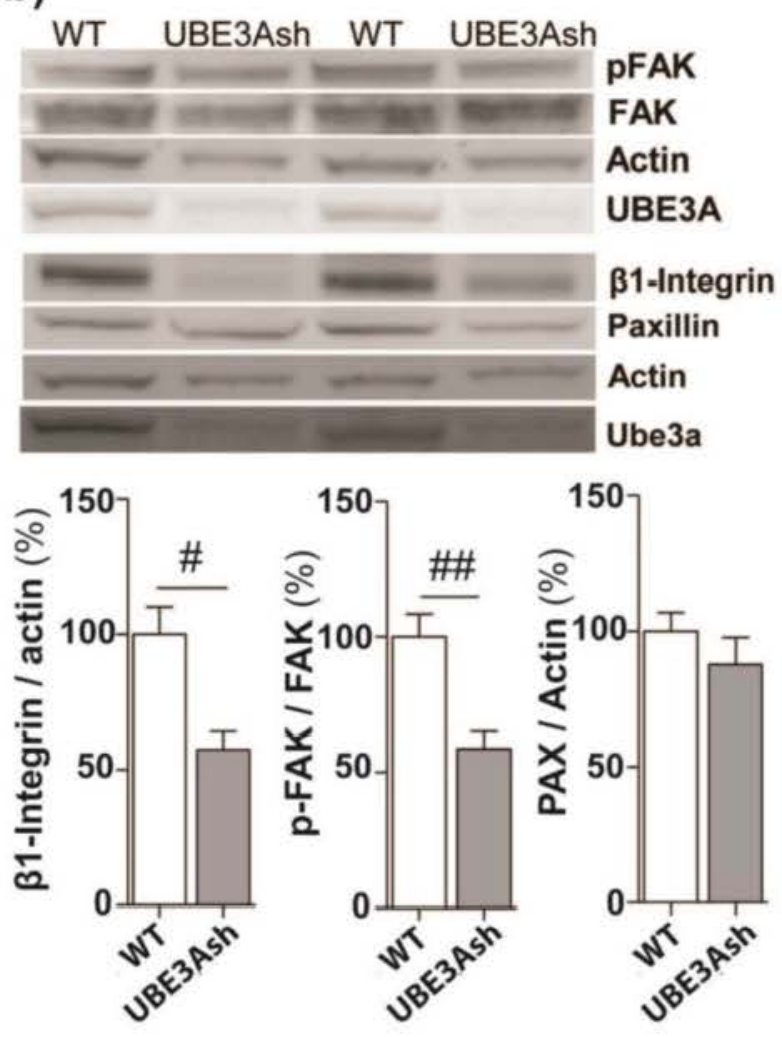

Figure 1. Adhesion and activation of FA pathway in UBE3Ash SHs. (a) SHs adhesion assay. The viability of adhered WT (white) and UBE3Ash (grey) SHs was quantified at 1-3-6 h and at $72 \mathrm{~h}$ on standard well plate and reported in \% with respect to the total number of seeded cells; \# P $<0.05$ WT vs. UBE3Ash, Student's t-test. (b) Representative Western-blot panels and blot analysis of $\beta 1$-integrin, phospho-FAK/FAK, and paxillin (e) levels, in WT (white) and UBE3Ash (grey) SHs cells. Results are reported in \% in respect to WT levels; \#/\#\# $\mathrm{P}<0.05 / 0.01 \mathrm{WT}$ vs. UBE3Ash, Student's $t$-test. no differences between WT and UBE3Ash SHs (not shown). Therefore the loss of UBE3A impairs FA alignment along GR tracks.

On GRs substrates, where a directional topographical information is present, usually FA maturation is favored by substrate directionality: this happened for both WT and UBE3Ash SHs (figure 2(i)). In fact, the size of aligned FAs was larger than the size of misaligned ones for both WT SHs $(\mathrm{P}<0.01, \mathrm{Al}$ vs. Misal) and UBE3Ash SHs, even though to a smaller extent $(\mathrm{P}<0.05$, Al vs. Misal; figure 2(i)).

We further analyzed FAs by sorting them into three size categories. This size distribution revealed a marked variation in the case of aligned FAs. Interestingly, for aligned FAs, UBE3Ash SHs showed an increase in the small-FA population $(\sim 45 \%$ in front to a $33 \%$ in WT; $\mathrm{P}<0.01$ WT $v$ s. UBE3Ash), and a reduction of large FAs (from $\sim 35 \%$ in WT to $25 \%$; $\mathrm{P}<0.05 \mathrm{WT}$ vs. UBE3Ash) (figure $2(1)$ ), similarly to what found also on isotropic standard surfaces. No evident trends were instead identified for misaligned FAs (figure $2(\mathrm{~m})$ ).

Overall, FA maturation (size) and spatial organization were affected by UBE3A silencing in SHs neuronal cells. In summary, these experiments demonstrated that UBE3A is needed for proper FA maturation, and is particularly important for the topographical sensing of FAs.

Because of the development of neurites is critically controlled by the maturation of FAs at their tip, we tested the guidance performance of SHs cells on GRs (Figure 3(a)). UBE3Ash SHs showed a reduced neurite alignment on T2 GRs $\left(16.6^{\circ} \pm 3^{\circ}\right)$ with respect to WT $\left(9.4^{\circ} \pm 1^{\circ}\right)(\mathrm{P}<0.05$ WT vs. UBE3Ash), as expected (Figure $3(\mathrm{~b})$ ). No effects on neurite development (i.e. length, number/cell) were induced by UBE3A silencing or by GRs pattern itself (results not shown). SHs were also further neuro-differentiated, leading to the formation of complex networks.: briefly, after $24 \mathrm{~h}$ from seeding, further neuronal differentiation was induced by treatment with retinoic acid $10 \mu \mathrm{M}$ in medium supplemented with $2 \%$ FBS for $6 \mathrm{~d}$ and then with BDNF $50 \mathrm{ng} \mathrm{mL}-1$ in medium supplemented with $0.5 \%$ FBS for additional $3 \mathrm{~d}$, as previously reported in [16]. The interaction with GRs induced high polarization and directional spatial arrangement, regardless of the SH subtype (figure S1(a), (b) (available online at https://stacks.iop.org/NANO/32/025708/mmedia)); in differentiated conditions the activation of FAK was still impaired (Figure S1(c)).

Considering the importance of the different isoforms in the UBE3A functioning and the only partial rescue of the UBE3A isoforms 2 and 3 on the axonal guidance in UBE3Adeficient neurons [35], we checked the possible influence of a non-coding UBE3A isoform in the contact guidance process. We measured in our SH cells the levels of miR-134, the competing endogenous RNA target of UBE3A isoform 1 in mice. However, miR-134 showed similar levels in both WT and UBE3Ash SHs (figure 3(c)), suggesting that UBE3A isoform 1 has likely not a primary role in the impaired FAs/neurite guidance of UBE3Ash SHs cells. 

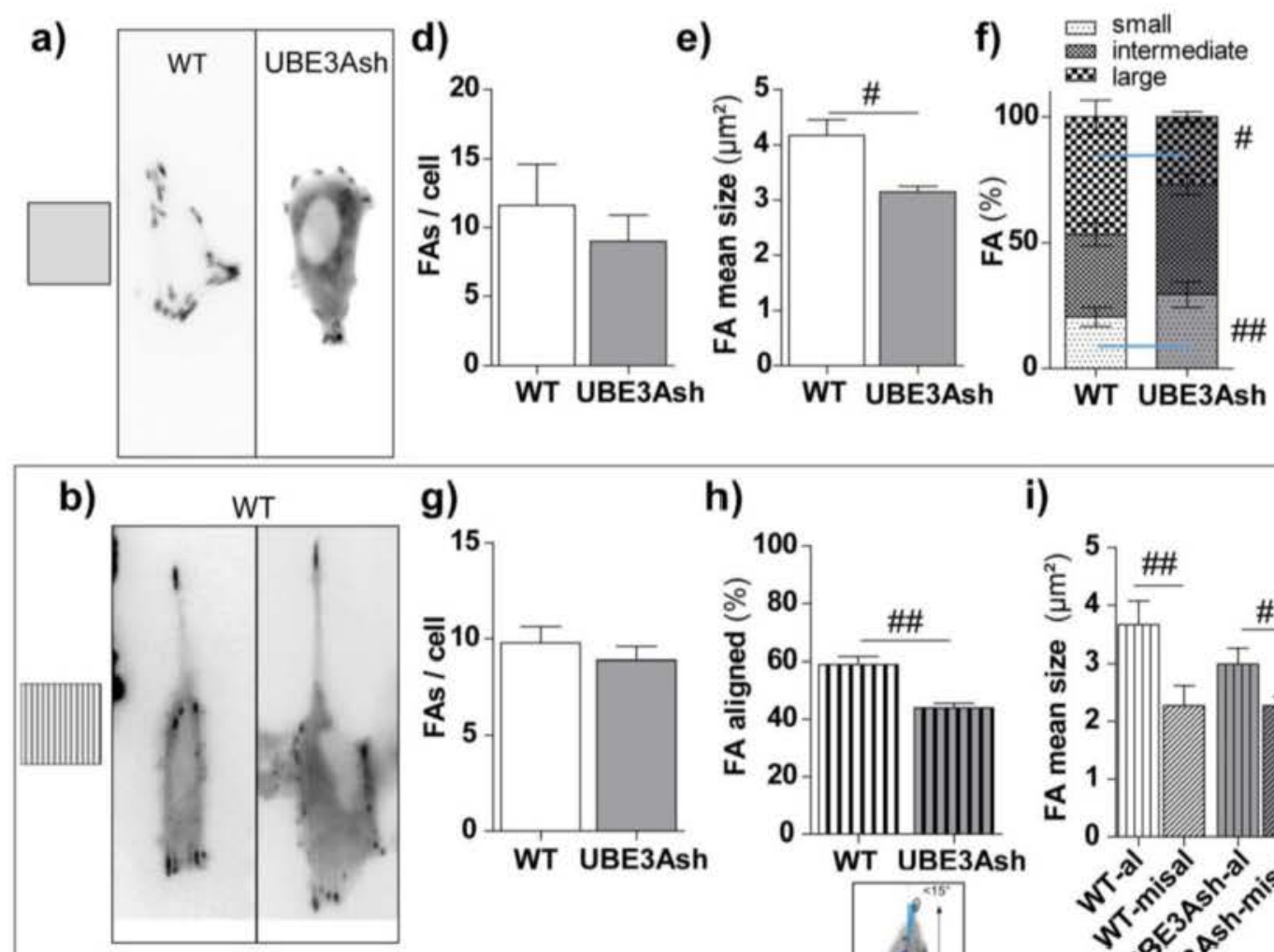

g)

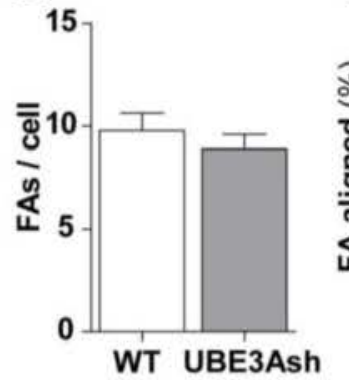

h)

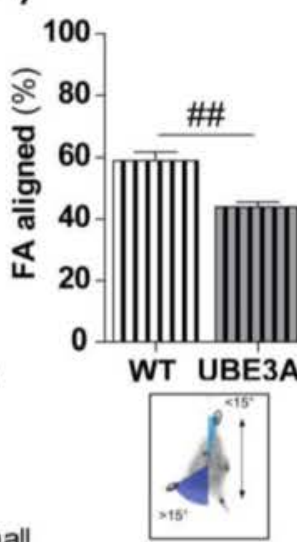

i)

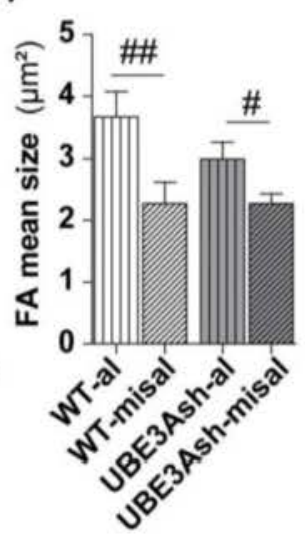

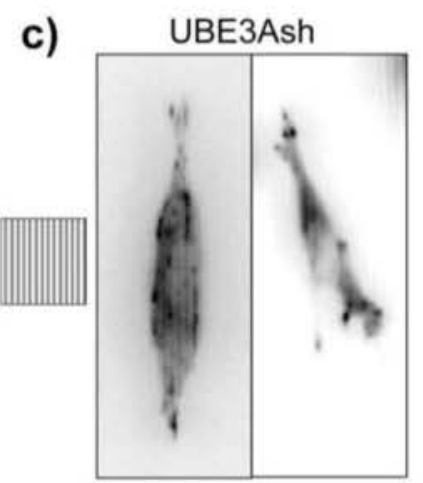
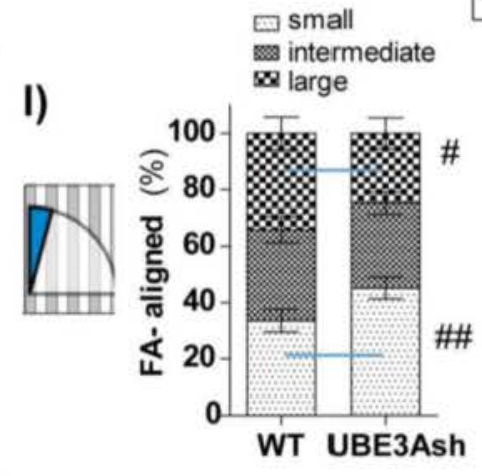

m)

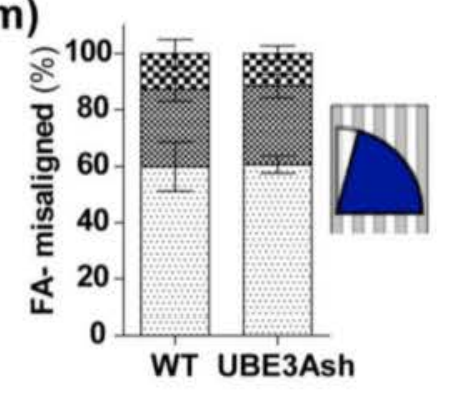

Figure 2. Impact of the loss of UBE3A on FA assembly and spatial distribution in SHs neuronal cells. (a)-(c) EGFP-Paxillin rich FAs in WT and UBE3Ash SHs on Flat (a) or on GRs (b), (c) imaged by TIRF microscopy with a laser penetration depth of $200 \mathrm{~nm}$. (d), (e), (f) FAs on flat substrates: (d) The number of FAs per cell; (e) The FA mean size ( $\mu \mathrm{m} 2)$ : \# P $<0.05$ WT vs. UBE3Ash, Student's $t$-test; (f) The distribution of FAs size: the \% of small (area $\left.\leq 2 \mu \mathrm{m}^{2}\right)$, intermediate $\left(2<\right.$ area $\left.\leq 4 \mu \mathrm{m}^{2}\right)$ and large $\left(\operatorname{area}>4 \mu \mathrm{m}^{2}\right)$ FAs is reported in small, medium, big dotted columns, in WT (white) and UBE3Ash (grey) cells; \#\# $\mathrm{P}<0.01 \mathrm{WT}$ vs. UBE3Ash for small FAs, \# $\mathrm{P}<0.05 \mathrm{WT}$ vs. UBE3Ash for large FAs, Student's $t$-test. (g)-(m) FAs on directional GRs substrates: (g) Number of FAs per cell. (h) FA alignment, reported as the $\%$ of FAs with alignment $\leq 15^{\circ}$ vs. GRs: \# P $<0.05$ WT vs UBE3Ash, Student's $t$-test; inset: Measurement of FA spatial distribution: FAs are identified as black spots and their radial position measured with respect to the GR direction. (i) The FA mean size $\left(\mu \mathrm{m}^{2}\right)$ : FAs with alignment $\leq 15^{\circ}$ were considered as aligned (al); FAs with alignment between $15^{\circ}$ and $90^{\circ}$ were considered as misaligned (misal): \#/\#\# $\mathrm{P}<0.05 / 0.01 \mathrm{Al} v s$. Misal for UBE3Ash and WT, respectively, Student's $t$-test. (I), (m) Size distribution of aligned $\left(0-15^{\circ}\right)(\mathbf{I})$ and misaligned $\left(15-90^{\circ}\right)(\mathbf{m})$ FAs for WT and UBE3Ash SHs; the $\%$ of small $\left(\right.$ area $\left.\leq 2 \mu \mathrm{m}^{2}\right)$, intermediate $\left(2<\right.$ area $\left.\leq 4 \mu \mathrm{m}^{2}\right)$ and large (area $>4 \mu \mathrm{m}^{2}$ ) FAs is reported in dotted-scale. (I) \#\# P $<0.01$ WT vs. UBE3Ash for small FAs-aligned, \# $\mathrm{P}<0.05$ WT vs. UBE3Ash for large FAs-aligned, Student's $t$-test.

Overall, the impaired neurite contact guidance of UBE3Ash SHs cells on GRs well correlates with the UBE3Ash FAs maturation and guidance deficits. miR-134 levels are not influenced by UBE3A loss in human neuronal SHs cells, suggesting that its activity is not crucial for FA and neurite development. 


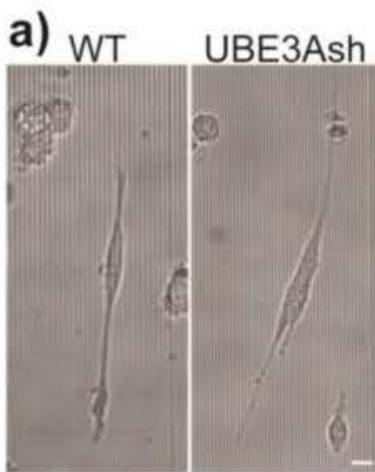

b)

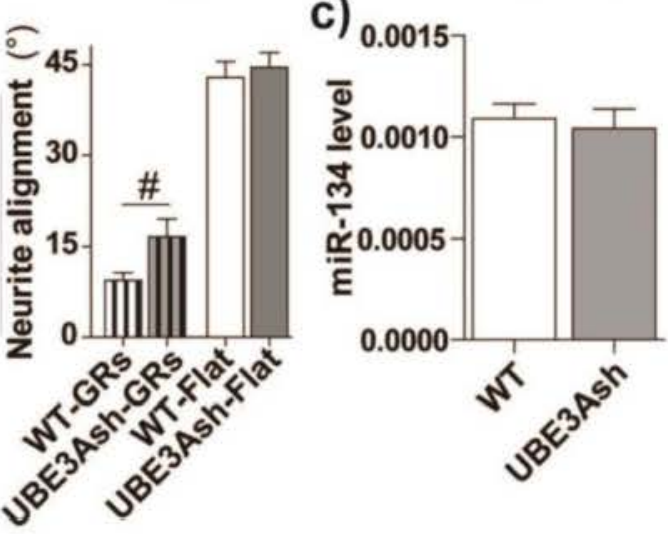

Figure 3. (a) Bright-field images (right) of WT and UBE3Ash SHs cells cultured on GRs; scale bar $=10 \mu \mathrm{m}$. Confocal images (left) of WT and UBE3Ash SHs neurites on GRs, immunostained for Tubulin-BIII (green) and actin fibers (red); inset: GRs pattern; scale bar $=10 \mu \mathrm{m}$. (b) Neurite alignment $\left({ }^{\circ}\right)$ on GR: neurite alignment is defined by approximating the neurite as a straight line from the initial to end point and taking the angle of this line versus the GR orientation (right panel). \# $\mathrm{P}<0.05 \mathrm{WT}$ vs. UBE3Ash on GRs, Student's $t$-test. (c) Measurement of miR-134 levels in WT (white) and UBE3Ash (grey) SHs cells; P > 0.05 Wilcoxon signed rank test, data $=$ mean \pm SD.

\subsection{Migration}

Considering that FAs are crucial for cell motion, we then investigated the migration performance of UBE3Ash SH cells. The ability of SHs to migrate was assessed first by single-cell migration experiments. SHs were cultured both on isotropic flat substrates and on GR lines and their motion was followed for $24 \mathrm{~h}$ by time-lapse bright-field microscopy (figure 4(a)).

Both WT and UBE3Ash SHs showed a directional migration on GRs. They migrated mainly parallel to the direction of the topography and the percentage of parallel steps (step with an angle within $15^{\circ}$ versus GR direction) was prevalent ( $\sim 60 \%$ of total steps) for both WT and UBE3Ash (figure 4(b)); conversely, the migration perpendicular to the pattern was highly suppressed on GRs $(\sim 5 \%)$. On FLAT migration was random, with no overall directional preference (i.e. for random motion with an arbitrary reference direction, the percent of aligned steps would theoretically be $20 \%$ ).
The mean overall migration speed was nearly the same on FLAT ( $\mathrm{V}=29 \pm 3$ and $26 \pm 4 \mu \mathrm{m} \mathrm{h}^{-1}$ for WT and UBE3Ash, respectively) and GR substrates $(V=35 \pm 7$ and $36 \pm 5 \mu \mathrm{m} \mathrm{h}^{-1}$ for WT and UBE3Ash, respectively) (figure 4(c)). In order to further describe the directional nature of single-cell migration, we selectively analyzed the velocity in its perpendicular and parallel components $\left(\mathrm{V}_{\perp}\right.$ and $\mathrm{V}_{\|}$, respectively). Both $\mathrm{SHs}$ populations showed enhanced $\mathrm{V}_{\|}$ and reduced $\mathrm{V}_{\perp}: \mathrm{V}_{\|}$was greater than $\mathrm{V}_{\perp}$ both for WT and UBE3Ash $\left(\mathrm{P}<0.05 \mathrm{~V}_{\|}\right.$vs. $\mathrm{V}_{\perp}$ and $\mathrm{V}$-GRs vs. $\mathrm{V}_{\perp}$; one-way ANOVA) (figure 4(c)).

We finally quantified the total cell displacement from $t=0$ position after $24 \mathrm{~h}(\mathrm{R})$ and also here WT and UBE3Ash SHs showed similar performances (figure 4(d)).

No defects in single cell motion have been found in both isotropic and anisotropic substrates. Altogether, these data demonstrate that UBE3Ash SHs cells are able to follow the directional stimulus of GRs and can migrated along tracks, as for WT SHs, regardless of their impaired FA maturation.

The ability of SHs to collectively migrate towards an open space was further assessed by wound-healing in vitro experiments. Gaps were created in SHs monolayers perpendicularly to the GRs and along a random direction on FLAT surfaces (figure 5(a)). As shown in figure 5(b), the woundhealing showed a slightly faster trend in WT with respect to UBE3Ash SHs $(\mathrm{P}<0.05$ WT vs. UBE3Ash at $48 \mathrm{~h}$ ). Interestingly, wound closure proceeded similarly on GRs and flat substrates. WT and UBE3Ash SHs showed also a similar energetic needs, with similar levels of glucose uptake (figure S2).

These data indicate a small delay of UBE3Ash cells in collective migration and wound-healing performance, at $48 \mathrm{~h}$ time point.

For a deeper understanding of the mechanisms of collective cell migration, we evaluated the contact-interactions in SHs cell monolayers. SHs monolayers were immunostained for $\mathrm{N}$-Cadherin ( $\mathrm{N}-\mathrm{Cad}$ ) in order to evaluate $\mathrm{N}$-Cad expression as a marker of cell-cell contact-interaction level. Confocal imaging shows that $\mathrm{N}-\mathrm{Cad}$ was mainly localized at cell periphery and at cell-cell junctions (figure 5(c)). The expression of $\mathrm{N}$-Cad appeared qualitatively indistinguishable between WT and UBE3Ash.

\section{Discussion}

Here, we investigated the adhesion and migration processes in an UBE3A-silenced neuronal cell model in vitro, by exploiting micro-grooved substrates. We exploited micro-gratings allowing the in vitro examination of specific topographical directional stimuli for cells. We analyzed the development of FAs by live-cell Total-Internal-Reflection-Fluorescence microscopy, and FA pathway at molecular level. We show that UBE3A-silenced SHs cells have impaired FA development, sensing and pathway activation, leading to a delayed adhesion and to a defective contact guidance in response to directional topographical stimuli. However, the overall migration behavior of UBE3A-silenced SHs is almost normal, in terms of 
a)
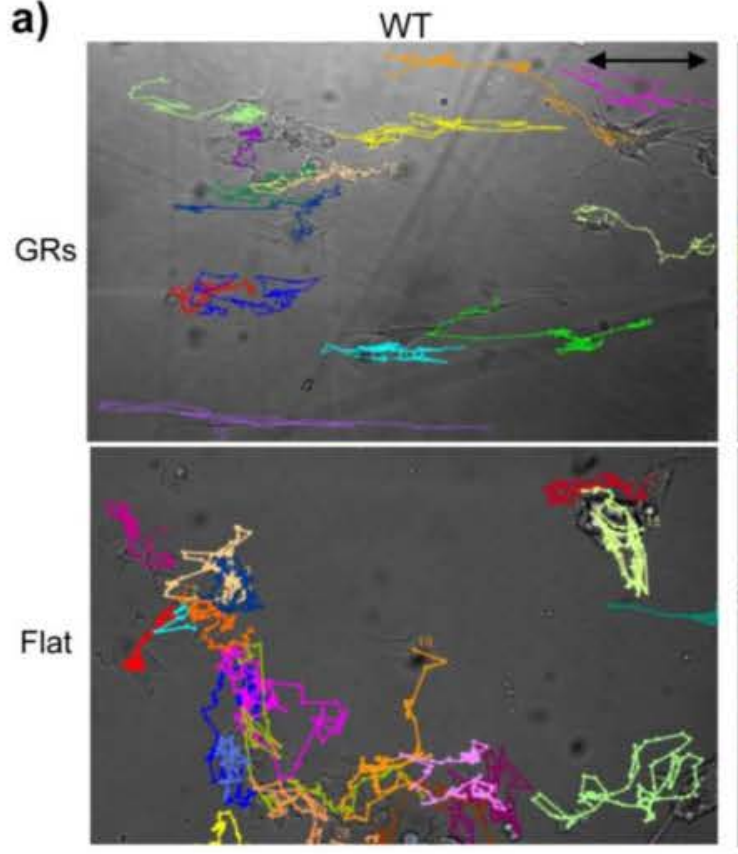

b)

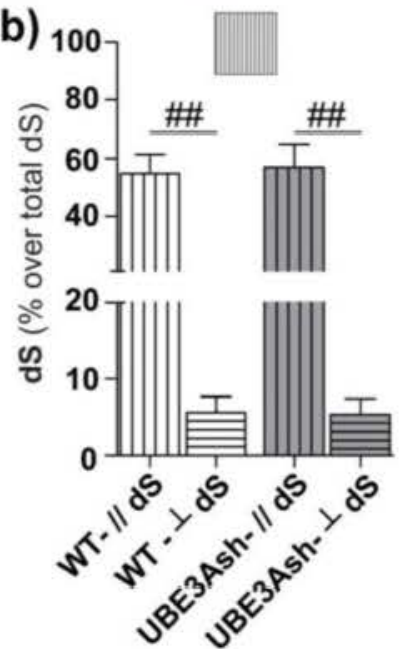

c) 50

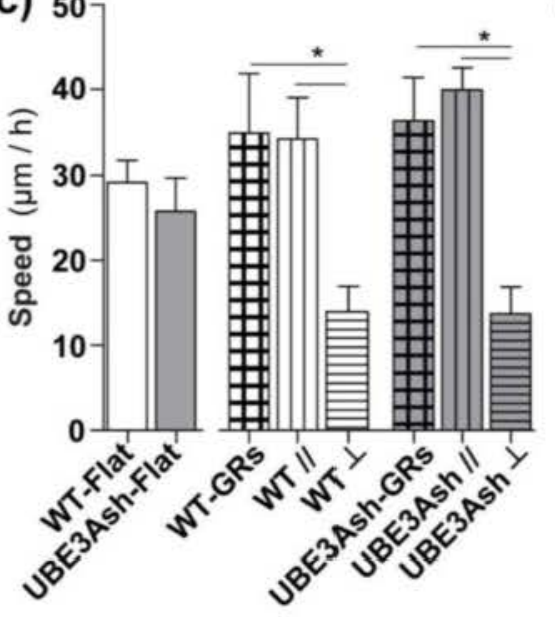

UBE3Ash
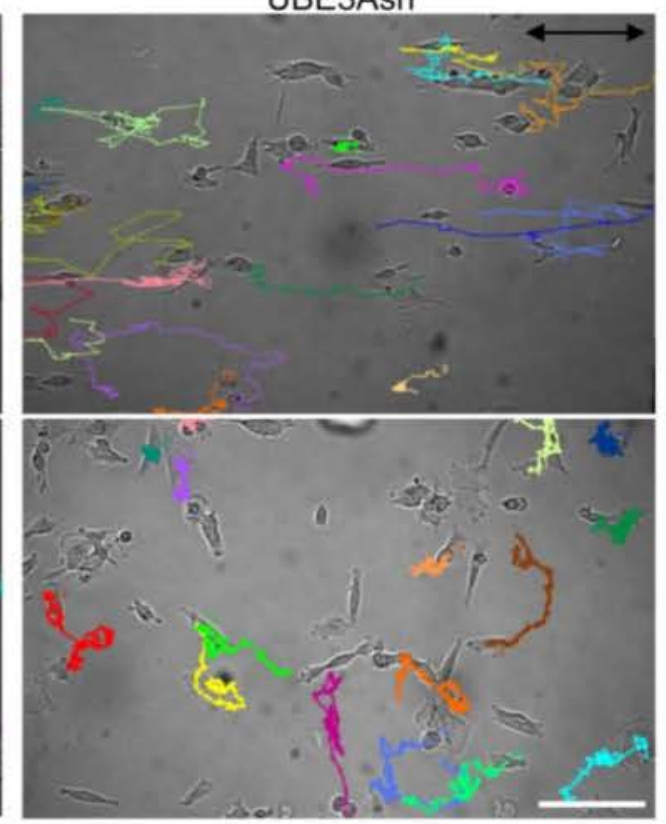

d)

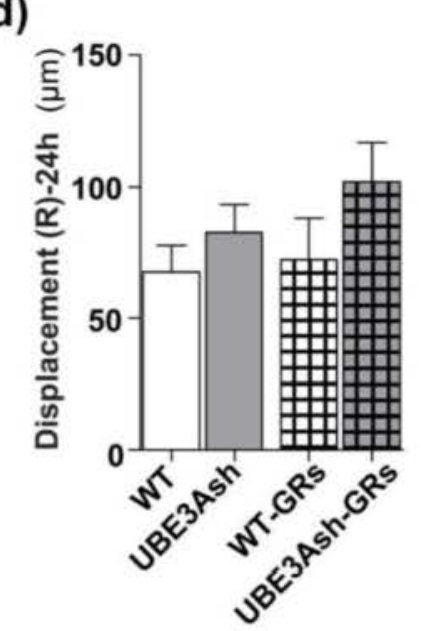

Figure 4. Single cell migration analysis. (a) Representative bright-field images of SHs migration patterns (colored tracks) on GRs and FLAT substrates. Black arrows represent the pattern direction; scale bar $=100 \mu \mathrm{m}$. (b) Percentage of parallel dS//and perpendicular dS $\perp$ steps to the pattern orientation for each SHs type on GRs: \#\# P $<0.01 \mathrm{dS} / / \mathrm{vs}$. dS $\perp$, Student's $t$-test. (c) Mean speed of migration of WT (white) and UBE3Ash (grey) SHs on Flat and GRs substrates: for SHs on GRs (left side), the mean parallel//and perpendicular $\perp$ speed in respect to GRs orientation are also reported; ${ }^{*} \mathrm{P}<0.05$ Speed//vs. $\perp$, and GRs vs. $\perp$, one-way ANOVA-Tukey's test-within each SHs type. (d) SHs final displacement R (at $t=24 \mathrm{~h}$ ) from the $t=0$ position for WT and UBE3Ash SHs on different substrates.

speed and directionality along GRs, except than for a small delay in collective migration.

Our previous results [35] suggested the presence of defects in the guidance and cytoskeleton dynamics in AS neurons. Here, for the first time we have investigated the adhesion process and the maturation of FAs in an AS-like neuronal model. The adhesion process itself is delayed (slowed down at early stages after seeding) in UBE3Ash SHs cells, and the FA pathway is impaired at the molecular level of FAK activation and $\beta 1$-Integrin expression.
We know that the adhesion process is mediated by the formation and maturation (i.e. enlargement) of FAs. We therefore here investigate FA development and in particular, we demonstrate an impaired FA maturation and topographical sensing by silencing UBE3A. In fact, UBE3A loss leads to a marked increase of the small-FA population, and to a reduction of large FAs, without affecting FA density, on flat isotropic substrates. Importantly, on GRs this happens only for the aligned FAs, while misaligned FAs are unperturbed in size, in agreement with our similar previous studies [14]. The impact of 
a)
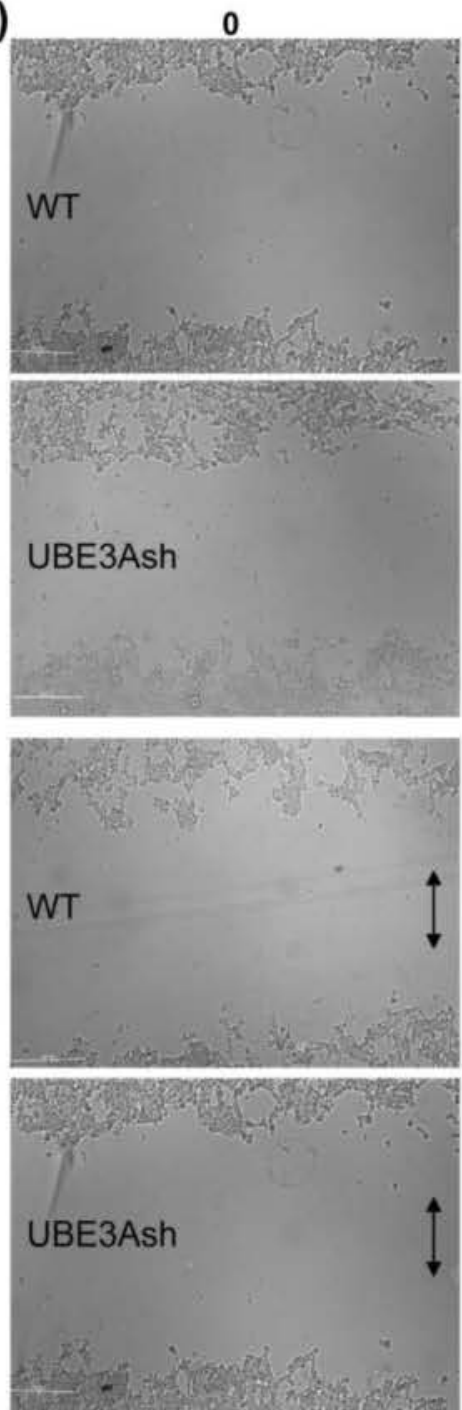

b)

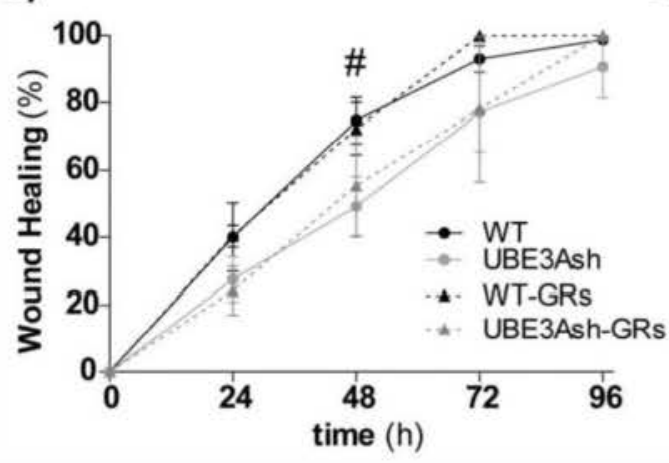

$24 \mathrm{~h}$
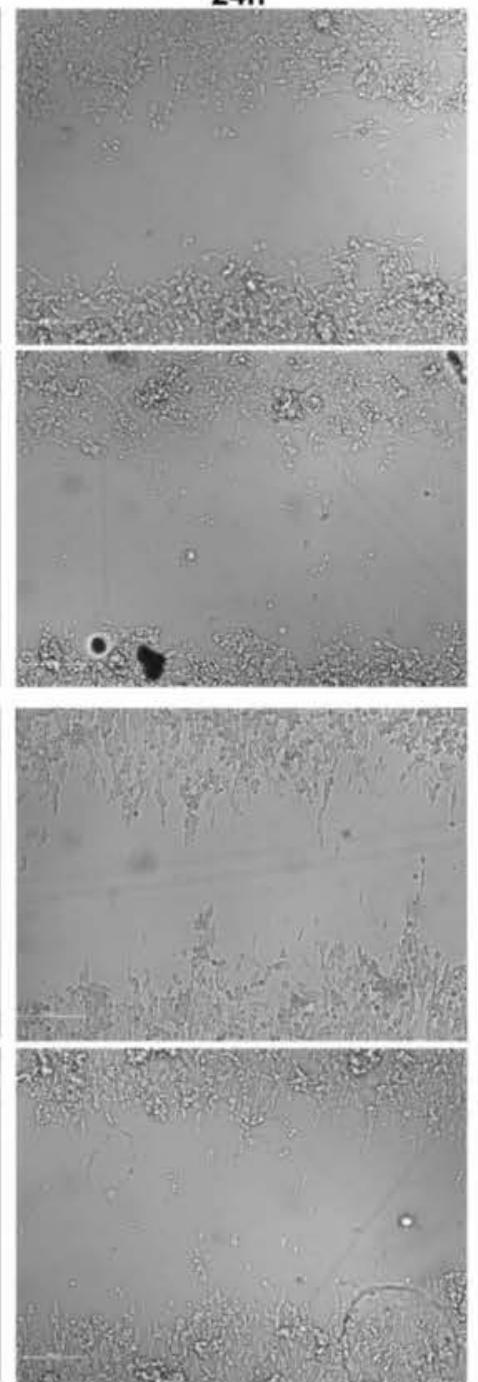

c)

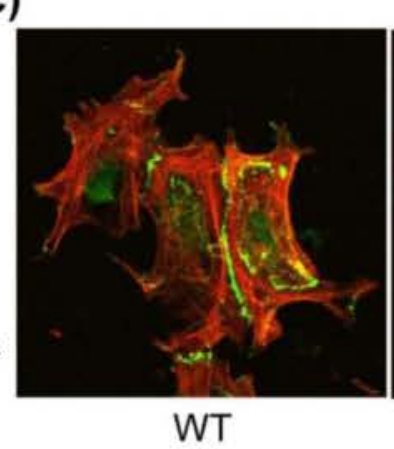

$72 \mathrm{~h}$
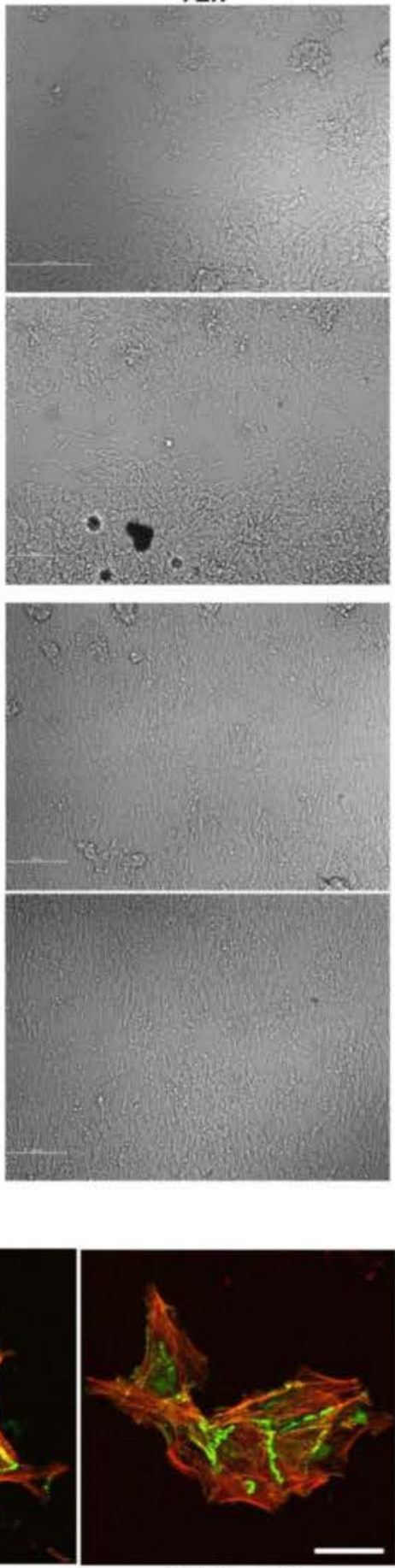

UBE3Ash

Figure 5. SHs collective migration. (a) Representative bright-field images of SHs collective migration on FLAT and GRs substrates, at $t=0-24-72 \mathrm{~h}$; black arrows $=$ GR direction; scale bars $=200 \mu \mathrm{m}$. (b) Wound closure $(\%)$ in time (h): \# P $<0.05 \mathrm{WT}$ vs. UBE3Ash, Student's $t$-test. (c) Confocal images of WT and UBE3Ash SHs grown on FLAT and immuno-fluorescently labeled for N-Cad (green) and actin fibers $(r e d)$; scale bar $=20 \mu \mathrm{m}$. 
UBE3A has been investigated taking advantage of the directional stimulus provided by the GR scaffolds, which are able to induce a visible phenotype in UBE3A-deficient neuronal cells $[34,35]$. In this framework, FAs act as topographical sensors on GRs. The loss of UBE3A impairs FA alignment along GR tracks, leading to a reduction of the FAs aligned to the pattern. As expected, this phenomenon correlates with a deficient neurite guidance along GRs. We know, from previous works, that the reduced maturation of aligned FAs leads to a reduced alignment of neurites along GRs [14]. The enlargement of the aligned FAs is favored on GRs and the mean size of aligned FAs is usually larger than the mean size of misaligned ones, for WT cells. Here, we found that this happen also for UBE3Ash SHs, even though to a smaller extent, thus suggesting that UBE3Ash cells can roughly read the topographical information. Overall, FAs establishment and maturation are compromised by the UBE3A loss and lead to the deficient neurite contact guidance in UBE3A-deficient neuronal cells.

Recently, it is emerging the role of the various UBE3A isoforms [30], which showed different localization and activity $[23,30,31,35]$. A strong correlation between the ASassociated mutations and the loss of UBE3A ligase activity (i.e. isoforms 2 and 3) has been demonstrated [41], however we showed that the reinstatement of both UBE3A isoforms 2 and 3 (catalytically active) in AS murine neurons at a very early stage of development only partially ameliorated their defective axonal guidance on GRs (where the treatment with a drug acting on the cytoskeleton, nocodazole, was successful) [35]. In mice, UBE3A isoform 1 has no ligase activity, but is involved in the control of neuronal arborization through the regulation of the microRNA miR-134 [31]. Importantly, miR134 also acts by interfering with several FA adhesion/cytoskeleton signaling effectors in different cells [33]. Beside this, the levels of miR-134 have not been investigated in AS neuronal models, at the best of our knowledge. Although less is known about the human UBE3A isoforms, it has been suggested that regulation of UBE3A- miRNA-134 might be conserved in mammals [31]. Therefore, here we looked at the possible contribution of miR-134, with the aim to understand the eventual molecular link between UBE3A and the deregulated guidance and FAs processes. However, the level of miR-134 was similar in WT and UBE3Ash SHs cells, suggesting that it has likely not a prominent role in the cell guidance/adhesion defects of human UBE3Ash SHs neuronal cells.

Considering the prominent role of FAs in driving cell motion [12], we investigated cell migration, both at single and a collective cell levels, and, in particular, the ability of GRs patterns to control and direct SHs cell migration. Our data show that single SHs migration is oriented along the GR lines, with the same efficiency for both WT and UBE3Ash SHs cells. They both migrated mainly parallel to the direction of the topography, and with similar speed and total displacement from the origin. Collective migration upon cell gaps was instead slightly impaired for UBE3Ash SHs on GRs, in particular at $48 \mathrm{~h}$ time point, but with a final closure of the gap within $96 \mathrm{~h}$ similar to WT. We also checked the cohesiveness of the cell-cell contacts by looking at N-Cadherin expression by fluorescence microscopy. N-Cadherins have a primary role in cell polarization, collective migration and control of axonal guidance [40, 42]. However, we found no major differences between WT and UBE3Ash SHs. All together, these data demonstrate that UBE3Ash SHs cells are able to migrate and to follow the main directional stimulus of GRs, as for WT SHs, regardless of their impaired FA maturation. Considering the much more complex environment that neurons find in vivo, we cannot exclude that the deficits of UBE3Ash cells in FA maturation and topographical sensing may have not patho-physiological implications and have no effects on the migration and guidance performance in AS conditions. In fact, it has been suggested that UBE3A could be involved in neuronal wiring $[19,43]$, because of UBE3A gene reinstatement in adult mice rescued electrophysiological properties but did not result in a behavioural rescue.

We hypothesized that the deficits in contact guidance of AS neuronal cells may be linked, directly or indirectly, to deregulations at the level of cytoskeleton dynamics. Several UBE3A targets converge on the regulation of cytoskeleton pathways [28], and we also demonstrated that nocodazole, a drug acting on cell contractility, is able to restore the deficit in axonal topographical guidance in AS neurons [35]. We now show that UBE3A loss impairs FAs sensing in a neuronal cell model, which then leads to the deficient contact guidance of neurites. However, the direct link between UBE3A and FA/cytoskeleton pathways needs to be investigated deeply to understand if it is driven by the ligase activity on some UBE3A-targets or by the nuclear factor activity of UBE3A.

Concluding, the loss of UBE3A affects the adhesion process in SH neuronal cell model in vitro. Our results demonstrated the use of GRs as advanced tools for testing the molecular mechanisms of cell contact guidance and shaping/migration dynamics in neuro-pathological models. Thanks to micro-structured GR substrates, we identified deficits in the maturation and topographical sensing processes of FAs in UBE3Ash neuronal cell model, which then leads to and explain the known impaired neurite contact guidance. The cell migration behavior of AS-like neuronal model is, however, mainly intact in vitro, in terms of directionality and speed, except than for a slight delay in the collective migration performance. The present results indicate the importance of further future investigations about the migration of UBE3Ash cells in more complex environments (e.g. in the presence of noise or of biochemical stimuli in vitro; in vivo, by in utero electroporation experiments) and about the functioning of FAs in UBE3A-null conditions, considering their crucial role at the synapses [44].

\section{Acknowledgments}

This work was supported by: Fondazione Umberto Veronesi with Post-doctoral Fellowship 2015 (project nano2RUN, to IT) and Post-doctoral Fellowship 2016 (project RE-guide, 
to IT); H2020 MSCA-Individual Fellowship-2017 grant Neuroguide (\#795948) to IT.

\section{ORCID iD}

I Tonazzini $\odot$ https://orcid.org/0000-0002-7323-2174

\section{References}

[1] Arimura $\mathrm{N}$ and Kaibuchi K 2007 Neuronal polarity: from extracellular signals to intracellular mechanisms Nat. Rev. Neurosci. $8194-205$

[2] Myers J P, Santiago-Medina M and Gomez T M 2011 Regulation of axonal outgrowth and pathfinding by integrin-ECM interactions Dev. Neurobiol. 71 901-23

[3] Geiger B. Spatz J P and Bershadsky A D 2009 Environmental sensing through focal adhesions Nat. Rev. Mol. Cell Biol. $1021-33$

[4] Navarro A I and Rico B 2014 Focal adhesion kinase function in neuronal development Curr. Opin. Neurobiol. 27 89-95

[5] Valiente M, Ciceri G, Rico B and Marín O 2011 Focal adhesion kinase modulates radial glia-dependent neuronal migration through connexin-26 J. Neurosci. 31 11678-91

[6] Baudry M and Bi X 2013 Learning and memory: an emergent property of cell motility Neurobiol. Learn. Mem. 104 64-72

[7] Myers J P and Gomez T M 2011 Focal adhesion kinase promotes integrin adhesion dynamics necessary for chemotropic turning of nerve growth cones J. Neurosci. 31 13585-95

[8] Stevens M M and George J H 2005 Exploring and engineering the cell surface interface Science $3101135-8$

[9] Tonazzini I and Cecchini M 2017 Neuronal Mechanisms for Nanotopography Sensing. Ebook Frontiers in Nanomedicine: Nanomedicine and Neurosciences: Advantages, Limitations and Safety Aspects Vol. 2 (Emirate of Sharjah: Bentham Science Publishers) pp 107-21

[10] Ferrari A, Cecchini M. Dhawan A, Micera S, Tonazzini I, Stabile R, Pisignano D and Beltram F 2011 Nanotopographic control of neuronal polarity Nano Lett. 11 505-11

[11] Ferrari A, Cecchini M, Beltram F and Degl'Innocenti R 2009 Directional PC12 cell migration along plastic nanotracks IEEE Trans. Biomed. Eng. 56 2692-6

[12] Ferrari A, Cecchini M, Serresi M, Faraci P, Pisignano D and Beltram F 2010 Neuronal polarity selection by topography-induced focal adhesion control Biomaterials 31 4682-94

[13] Tonazzini I, Pellegrini M, Pellegrino M and Cecchini M 2014 Interaction of leech neurons with topographical gratings: comparison with rodent and human neuronal lines and primary cells Interface Focus $420130047-20130047$

[14] Tonazzini I, Meucci S, Faraci P. Beltram F and Cecchini M 2013 Neuronal differentiation on anisotropic substrates and the influence of nanotopographical noise on neurite contact guidance Biomaterials 34 6027-36

[15] Ferrari A, Faraci P, Cecchini M and Beltram F 2010 The effect of alternative neuronal differentiation pathways on $\mathrm{PC} 12$ cell adhesion and neurite alignment to nanogratings Biomaterials 31 2565-73

[16] Tonazzini I, Cecchini A, Elgersma Y and Cecchini M 2014 Interaction of SH-SY5Y cells with nanogratings during neuronal differentiation: comparison with primary neurons Adv. Healthcare Mater. 3 581-7

[17] McFadden K and Minshew N J 2013 Evidence for dysregulation of axonal growth and guidance in the etiology of ASD Frontiers Hum. Neurosci. 7671
[18] Kawabe $\mathrm{H}$ and Brose $\mathrm{N} 2011$ The role of ubiquitylation in nerve cell development Nat. Rev. Neurosci. 12 251-68

[19] Silva-santos S, Van Woerden G M, Bruinsma C F and Mientjes E 2015 Ube3a reinstatement identifies distinct treatment windows in Angelman syndrome model mice J. Clin. Invest. 125 2069-76

[20] Jiang Y H, Armstrong D, Albrecht U, Atkins C M, Noebels J L, Eichele G, Sweatt J D and Beaudet A L 1998 Mutation of the Angelman ubiquitin ligase in mice causes increased cytoplasmic p53 and deficits of contextual learning and long-term potentiation Neuron 21 799-81 I

[21] Elgersma Y 2015 A molecular tightrope Nature $52650-5$ ]

[22] Glessner J T et al 2010 NIH Public Access 459 569-73.

[23] Miao S, Chen R, Ye J, Tan G-H, Li S, Zhang J, Jiang Y-H and Xiong Z-Q 2013 The angelman syndrome protein ube $3 \mathrm{a}$ is required for polarized dendrite morphogenesis in pyramidal neurons J. Neurosci. 33 327-33

[24] Dindot S V, Antalffy B A, Bhattacharjee M B and Beaudet A L 2008 The Angelman syndrome ubiquitin ligase localizes to the synapse and nucleus, and maternal deficiency results in abnormal dendritic spine morphology Hum. Mol. Genet. $17111-8$

[25] Lu Y, Wang F, Li Y, Ferris J, Lee J-A and Gao F-B 2009 The Drosophila homologue of the Angelman syndrome ubiquitin ligase regulates the formation of terminal dendritic branches Hum. Mol. Genet. 18 454-62

[26] Baudry M et al 2012 Ampakines promote spine actin polymerization, long-term potentiation, and learning in a mouse model of Angelman syndrome Neurobiol. Dis. $47210-5$

[27] Jensen L, Farook M F and Reiter L T 2013 Proteomic profiling in Drosophila reveals potential dube 3 a regulation of the actin cytoskeleton and neuronal homeostasis PLoS One 8 e61952

[28] Sell G L and Margolis S S 2015 From UBE3A to Angelman syndrome: a substrate perspective Front. Neurosci. $91-6$

[29] Woerden G M, Van Harris K D, Hojjati M R, Gustin R M, Qiu S, Freire R D A, Jiang Y-H, Elgersma Y and Weeber E J 2007 Rescue of neurological deficits in a mouse model for Angelman syndrome by reduction of $\alpha \mathrm{CaMKII}$ inhibitory phosphorylation Nat. Neurosci. 10 280-2

[30] Avagliano Trezza R et al 2019 Loss of nuclear UBE3A causes electrophysiological and behavioral deficits in mice and is associated with Angelman syndrome Nat. Neurosci. 22 1235-47

[31] Valluy $\mathrm{J}$ et al 2015 A coding-independent function of an alternative Ube 3 a transcript during neuronal development Nat. Neurosci. 18 666-73 https://www.nature.com /doifinder/10.1038/nn.3996

[32] Han L et al 2011 Regulation of chemotropic guidance of nerve growth cones by microRNA Mol. Brain 440

[33] Poitz D M et al 2013 MiR-134-mediated $\beta 1$ integrin expression and function in mesenchymal stem cells Biochim. Biophys. Acta $\mathbf{1 8 3 3} 3396-404$

[34] Tonazzini I, Meucci S, Van Woerden G M M, Elgersma Y and Cecchini M 2016 Impaired neurite contact guidance in ubiquitin ligase E3a (Ube3a)-deficient hippocampal neurons on nanostructured substrates Adv. Healthcare Mater. $5850-62$

[35] Tonazzini I, Van Woerden G M, Masciullo C, Mientjes E J, Elgersma Y and Cecchini M 2019 The role of ubiquitin ligase E3A in polarized contact guidance and rescue strategies in UBE3A-deficient hippocampal neurons $\mathrm{Mol}$. Autism 10 1-18

[36] Cecchini M. Ferrari A and Beltram F 2008 PC12 polarity on biopolymer nanogratings J. Phys. Conf. Ser. 100012003

[37] Kuhnle S, Mothes B, Matentzoglu K and Scheffner M 2013 Role of the ubiquitin ligase E6AP/UBE3A in controlling 
levels of the synaptic protein Arc Proc. Natl Acad. Sci. $1108888-93$

[38] Tonazzini I, Masciullo C, Savi E, Sonato A, Romanato F and Cecchini M 2020 Neuronal contact guidance and YAP signaling on ultra-small nanogratings Sci. Rep. 103742

[39] Rao X, Huang X, Zhou Z and Lin X 2013 An improvement of the $2^{\wedge}$ (-delta delta CT) method for quantitative real-time polymerase chain reaction data analysis Biostat. Bioinform. Biomath. 3 71-85

[40] Tonazzini I, Jacchetti E, Meucci S, Beltram F and Cecchini M 2015 Schwann cell contact guidance versus boundary -interaction in functional wound healing along nano and microstructured membranes Adv. Healthcare Mater. $41849-60$

[41] Cooper E M, Hudson A W, Amos J, Wagstaff J and Howley P M 2004 Biochemical analysis of Angelman syndrome-associated mutations in the E3 ubiquitin ligase E6-associated protein J. Biol. Chem.

$27941208-17$

[42] Gärtner A, Fornasiero E F and Dotti C G 2015 Cadherins as regulators of neuronal polarity Cell Adhes. Migr. 9 175-82

[43] Rotaru D C, van Woerden G M, Wallaard I and Elgersma Y 2018 Adult Ube 3 a gene reinstatement restores the electrophysiological deficits of prefrontal cortex layer 5 neurons in a mouse model of angelman syndrome $J$. Neurosci. 38 8011-30

[44] Rico B, Beggs H E, Schahin-Reed D, Kimes N, Schmidt A and Reichardt L F 2004 Control of axonal branching and synapse formation by focal adhesion kinase Nat. Neurosci. 7 1059-69 